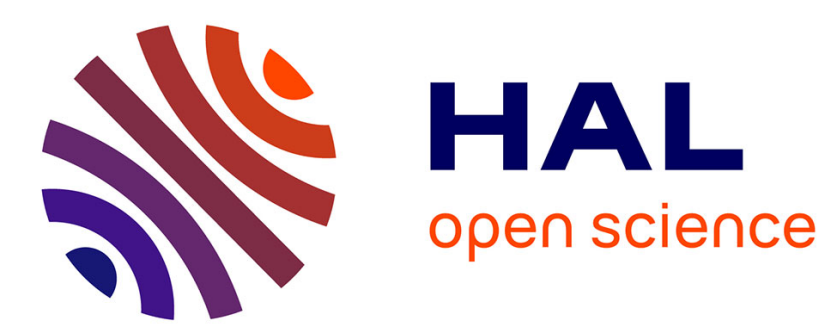

\title{
La fin du Mésolithique dans l'arc jurassien : approche statistique des industries lithiques taillées. Thomas Perrin
}

\section{To cite this version:}

Thomas Perrin. La fin du Mésolithique dans l'arc jurassien: approche statistique des industries lithiques taillées.. Bulletin de la Société préhistorique française, 2002, 99 (3), pp.487-499. hal00266590

\author{
HAL Id: hal-00266590 \\ https://hal.science/hal-00266590
}

Submitted on 14 Oct 2012

HAL is a multi-disciplinary open access archive for the deposit and dissemination of scientific research documents, whether they are published or not. The documents may come from teaching and research institutions in France or abroad, or from public or private research centers.
L'archive ouverte pluridisciplinaire HAL, est destinée au dépôt et à la diffusion de documents scientifiques de niveau recherche, publiés ou non, émanant des établissements d'enseignement et de recherche français ou étrangers, des laboratoires publics ou privés. 


\title{
La fin du Mésolithique dans l'arc jurassien : approche statistique Thomas PRRIN des industries lithiques taillées
}

\begin{abstract}
Résumé
Cet article propose une approche typologique renouvelée des industries lithiques mésolithiques de l'arc jurassien et de ses marges. Il se base sur les inventaires typologiques publiés des principaux sites stratifiés régionaux. Par le biais d'analyses statistiques multivariées, il est possible de proposer une sériation chronotypologique des gisements en quatre étapes principales. Celles-ci sont ensuite confrontées aux données du radiocarbone. Les perspectives culturelles sont ensuite abordées, pour les étapes récente et finale.
\end{abstract}

\begin{abstract}
This paper proposes a renewed typological approach to the Mesolithic industries of the Jura and its neighbouring regions. It is based on the typological inventories published for the main regional sites. By means of multivariate statistical analyses, it is possible to propose a chronotypological seriation of the layers in four main stages. These are then confronted with the radiocarbon data. The cultural prospects are then approached, for the recent and final stages.
\end{abstract}

\section{INTRODUCTION}

Dans un récent travail de thèse, nous avons eu l'occasion d'analyser les caractères évolutifs des industries lithiques taillées néolithiques du haut Bassin rhodanien (Perrin, 2001). Si l'ensemble de la période néolithique a été abordé, notamment à travers l'étude de la grotte du Gardon (Ambérieu-en-Bugey, Ain) fouillée par Jean-Louis Voruz, une partie importante de ce travail était orientée vers la mise en évidence et la compréhension des processus de néolithisation $\mathrm{du}$ haut Bassin rhodanien. Des résultats originaux ont pu être dégagés, puisqu'ils suggèrent, entre autres, la possible coexistence territoriale de deux populations distinctes dont les industries lithiques renvoient, pour l'une, au Mésolithique final jurassien, et, pour l'autre, au Néolithique ancien méridional (ibid. ; Perrin, à paraître). Si nous avons déjà pu présenter le "versant" néolithique des choses par ailleurs (ibid.), nous souhaitons dans ce court article présenter une approche actualisée des industries du Mésolithique jurassien, et notamment celles de la fin de cette période. En effet, aborder les processus de néolithisation ne peut se faire sans une bonne connaissance du milieu "d'impact" (qu'il soit colonisé ou acculturé). Or, régionalement, si les synthèses typologiques sont nombreuses (Thévenin, 1990, 1991, 1995 et 1998 ; FrelinKhatib et Thévenin, 2000, par exemple), l'approche détaillée des industries est rarement présentée. Dans le cadre de ce travail de thèse, nous avons donc cherché à formaliser l'évolution, notamment typologique, des industries de la fin du Mésolithique de l'arc jurassien et de ses marges. Cette démarche se base sur les inventaires typologiques publiés des principaux sites stratifiés régionaux. Ce sont les résultats de ces analyses qui sont présentés ici. 


\section{RAPPEL DU CADRE CHRONOCULTUREL DU CENTRE-EST DE LA FRANCE}

Traditionnellement, en France, le Mésolithique est découpé en trois phases : ancienne, moyenne et récente, tripartition à laquelle de plus en plus d'auteurs rajoutent une phase finale (par exemple Rozoy, 1978; Thévenin, 1998 ou Marchand, 1999). Ces subdivisions chronotypologiques sont essentiellement effectuées par "référence aux armatures microlithiques qui constituent les meilleurs marqueurs chronologiques et culturels" (Bintz et al., 1995, p. 41) ainsi que, dans une moindre mesure, sur l'évolution des "styles de débitage" (Rozoy, 1968 et 1978; Gob, 1976). Cette dernière notion renvoie à une "perception globale, esthétique" (Rozoy, 1978, p. 38) des industries. Elle correspond à une tentative de reconnaissance du fait technique par la caractérisation de certains aspects des produits obtenus, mais le plus souvent sans recherche des séquences opératoires (cf. pour un exemple contraire Walczak, 1998). L'aspect technologique mobilisé dans cette notion de style reste en réalité subordonné à une signification chronoculturelle ou anthropologique attribuée a priori. C'est donc une notion certes commode car synthétique, mais qui ne saurait suffire à caractériser précisément une industrie.

Quoi qu'il en soit, alors que le Mésolithique ancien et moyen se caractérise, dans cette région, par des industries sur lamelles irrégulières ("style de Coincy") et par des armatures en forme de triangle essentiellement, le Mésolithique récent correspond à une rupture technologique avec des lames plus régulières ("style de Montbani") et typologique avec des armatures en forme de trapèzes asymétriques (Thévenin, 1995, fig. 30 et 31). Le Mésolithique final se situe dans la continuité typologique du Mésolithique récent avec cependant l'apparition de la retouche inverse plate sur les armatures, qualifiées alors d'évoluées. La variété régionale de ces armatures correspond au type connu sous le nom de "pointe de Bavans" (Aimé et Jeunesse, 1986). Cette armature asymétrique à base concave se répartit sur l'ensemble de l'arc jurassien et du Plateau suisse (Löhr, 1991) et "il est généralement admis que les trapèzes à retouches inverses plates de la base appartiennent à un stade un peu plus récent que les trapèzes à troncature directe, bien que ces derniers perdurent" (ibid., p. 53).

Cette rupture très forte entre le Mésolithique ancienmoyen et le Mésolithique récent-final est parfois mise sur le compte d'influx provenant d'un Proche-Orient déjà néolithisé (Clark, 1958; Jeunesse, 1998; Bintz, 1999). Selon cette hypothèse, la diffusion de l'agriculture s'inscrirait alors au sein d'un réseau de relations déjà en place : "les indices précoces d'agriculture découverts ces dernières années redonnent [...] une actualité nouvelle à cette théorie quelque peu oubliée" (Jeunesse, 1998, p. 213). Celle-ci reste néanmoins extrêmement fragile car "les étapes de cette diffusion ne sont pas encore définies dans le détail. Une lacune considérable de recherche et de publication doit être constatée, plus particulièrement pour toute la zone entre le Caucase et le bord oriental de la mer d'Azov" (Mazurié de Keroualin, 2000, p. 199).

\section{MATIÈRES ET TECHNIQUES : ÉTAT DES CONNAISSANCES}

Du point de vue des matières premières, les gisements du Mésolithique récent et final jurassien livrent des industries principalement réalisées sur des matériaux locaux. Dans quelques cas cependant, comme à la baume de Montandon, "le silex local joue un rôle secondaire, alors que les silex d'une provenance supérieure à $20 \mathrm{~km}$ dominent largement et montrent une origine variée" (Cupillard, 1998b, p. 156). Les analyses de provenance des matières exotiques permettent à J. Affolter (2000) de dresser une esquisse des réseaux de relations ou d'échanges entre les groupes humains. Elle remarque aussi que les gisements jurassiens au sens strict (Baulmes, Mollendruz, Gripons, Bavans, Ranchot...) ne présentent "aucun lien clair avec les sites du plateau" (Affolter, 1998, p. 88). Ils montrent par contre "des relations ténues, ou du moins une certaine communauté de territoire" (ibid.).

D'un point de vue technologique, il a été montré l'utilisation de la percussion indirecte selon un rythme très régulier pour la production de lames (Séara, 1998 et 2000). Cette volonté de produire des lames régulières est attestée dans à peu près tous les gisements et constitue un des éléments distinctifs de ce Mésolithique récent (i.e. style Montbani). Les récentes études de F. Séara (1998) sur les gisements de Choisey et de Ruffey-sur-Seille montrent que le Mésolithique récent voit la dominance de plans de frappe facettés. Les nucléus y sont cannelés, à table laminaire souvent plate et dont le dos a été façonné soit par une crête postérieure, soit par une crête postéro-latérale. L'évolution du débitage entre Mésolithique ancien et moyen et Mésolithique récent "aboutit à un niveau de technicité suffisamment élevé pour assurer, même à partir de matière première de qualité médiocre, une production bien souvent régulière" (Séara, 1998, p. 152). Ces lames régulières sont ensuite fracturées par la technique du microburin pour la fabrication d'armatures.

En l'état des connaissances, il ne semble pas exister d'évolution technologique nette entre le Mésolithique récent et le Mésolithique final. Celui-ci ne s'individualiserait que par une évolution typologique.

\section{APPROCHE DE TYPOLOGIE STATISTIQUE}

\section{Méthode}

Réaliser une analyse statistique des données typologiques de la fin du Mésolithique implique de fusionner au sein d'une même typologie les divers sites retenus. Cela ne fut pas sans mal ni imperfections : les listes typologiques utilisées par les divers auteurs (dérivées de celles de J.-G. Rozoy, 1978 et G. Laplace, 1964 ou autres) proposent des types dont la correspondance n'est pas parfaite. Un type présenté dans l'une sera éclaté au sein de plusieurs dans l'autre et inversement. La liste typologique que nous avons utilisée est basée 


\begin{tabular}{|l|l|ll|}
\hline \multirow{2}{*}{ Groupes } & \multicolumn{1}{|c|}{ criteres distinetifs } & \multicolumn{2}{|c|}{ Classes } \\
\hline \multirow{2}{*}{ transversal abrupt } & \multirow{2}{*}{ une ou deux extrémités } & troncatures & TR \\
\cline { 3 - 4 } & & bitroncatures géométriques & BG \\
\hline \multirow{2}{*}{ transversal non abrupt } & & grattoirs & GR \\
\hline \multirow{2}{*}{ latéral abrupt } & & bords abattus abrupts & BA \\
\hline \multirow{2}{*}{ latéral non abrupt } & \multirow{3}{*}{ uni- ou bifaciales } & pièces bifaciales & RA \\
\hline \multirow{2}{*}{ spécial } & & technique du microburin & MB \\
\cline { 3 - 4 } & & burins et chanfreins & BC \\
\cline { 3 - 4 } & & coches clactoniennes & CC \\
\hline
\end{tabular}

Fig. 1 - Structuration typologique des classes d'outils au sein des groupes jes pièces retouchées.

sur celle de D. Binder (1987) adaptée au contexte chronoculturel régional (Perrin, 2001). Par la hiérarchisation des critères descriptifs, cette typologie permet la reconnaissance de familles d'outils, de groupes, de classes, de types et de sous-types. Trois familles d'outils ont ainsi été définies : les pièces retouchées, les pièces utilisées brutes et les blocs façonnés. Au sein des pièces retouchées, la localisation des retouches, leur inclinaison etc., permet de distinguer cinq groupes subdivisés en neuf classes (fig. 1). Chacune de ces classes est ensuite répartie en plusieurs types et parfois en sous-types. Ainsi, les armatures de flèches du Mésolithique récent et final appartiennent à la classe des bitroncatures géométriques ou à celle des bords abattus abrupts.

L'analyse statistique des sites du Mésolithique jurassien a pris pour base l'ensemble des outillages présents sur chaque gisement. Cependant, tous ne sont pas semblablement significatifs dans la recherche d'une structuration factorielle. Certains ont donc été écartés au cours de l'analyse ou agrégés avec d'autres types proches. En définitive, la structuration chronotypologique des industries ne mobilise que neuf types d'outils, et principalement des armatures. On trouve ainsi les lamelles étroites à un ou deux bords abattus, dégageant une extrémité aiguë. Ce type est codé en BA11, et comprend par exemple les pointes de Sauveterre. Le type BA12 ne diffère du précédent que par une base façonnée par une troncature directe. Ce type comprend par exemple les triangles de Montclus. Nous avons également retenu le type TR22, c'est-à-dire les pièces minces (épaisseur inférieure à $8 \mathrm{~mm}$ : Binder, 1987) portant une troncature normale, directe ou croisée. La catégorie générale des lames à un bord abattu a également été conservée (BA2), ainsi que celle des lames à enlèvements irréguliers (IR22 : dont les lamelles Montbani). Au sein des bitroncatures géométriques, nous avons mobilisé quatre types de pièces (fig. 2). Il s'agit tout d'abord des géométriques à troncatures obliques et piquant trièdre (BG11), qui peuvent présenter des retouches inverses rasantes (BG13) comme les pointes de Sonchamp par exemple (pointes triangulaires à retouches inverses rasantes; Rozoy,
1978, p. 64). Le groupe BG15-16 correspond aux pointes asymétriques, c'est-à-dire à grande troncature très oblique, dont la base concave est façonnée par des retouches bifaciales. Ce type rassemble la plupart des armatures à éperons, dont la célèbre pointe de Bavans. Enfin, la dernière catégorie est celle des trapèzes à deux troncatures directes, qui peuvent être asymétriques, F21) ou symétriques (BG22).

\section{Choix des sites}

Dans cette analyse, nous avons pris le parti de ne retenir que les gisements suivants (fig. 3) :

- abris de Bavans c. 9 à c. 5, Bavans, Jura (Aimé dir., 1993);

- Birsmatten Basisgrotte H5 à H1, Nenzlingen, Bâle Landschaft (Bandi, 1963; Rozoy, 1978);

- abri Freymond c. 4d et c. 4b, Mont-la-Ville, Vaud (Pignat et al., 1998);

- abri de la Grande Rivoire c. B3b, Sassenage, Isère (Picavet, 1991);

- abri des Gripons c. 4 et c. 3, Saint-Ursanne, Jura (Pouzas et al., 1991);

- Liesbergmühle VI KII, KIH2 et KIH1, Liesberg, Berne (Hofmann-Wyss, 1978);

- baume de Montandon c. VI et IV, Saint-Hippolyte, Doubs (Cupillard et al., 2000);

- abri du Roseau c. 3, Neuville-sur-Ain, Ain (Wittig, 1997);

- Schötz 7, Schötz, Lucerne (Wyss, 1979);

- grotte de Souhait c. 4, Montagnieu, Ain (Desbrosse et al., 1961);

- abri de Sous-Balme à Culoz c. 3 niv. 3 et niv. 1, et "Culoz abri", Culoz, Ain (Vilain, 1966);

- abri de la Touvière E2 à E4, Arbignieu, Ain (Morelon, 1973).

À l'exception de Schötz 7, ce sont tous des sites stratifiés. Ce choix d'une restriction aux stratigraphies nous permet de confronter l'adéquation de la structure obtenue par l'analyse statistique à celle de la réalité stratigraphique. Cette démarche nécessite donc de considérer de façon individuelle chacune des couches de 


\begin{tabular}{|c|c|c|c|c|}
\hline Type & Définition & Schéma type & Sous-types & $\begin{array}{l}\text { Correspondance } \\
\text { D. Binder } 1987\end{array}$ \\
\hline $\begin{array}{l}\text { BG11 - géom. à } \\
\text { tronc. obliques et } \\
\text { piquant-trièdre }\end{array}$ & $\begin{array}{l}\text { bitronc. géo. dont } \\
\text { une tronc. au } \\
\text { moins porte une } \\
\text { facette de piquant- } \\
\text { trièdre }\end{array}$ & & $\begin{array}{l}\text { BG11A - trapèze } \\
\text { BG11B - triangle }\end{array}$ & $\begin{array}{c}\text { BG11 } \\
(\mathrm{A}, \mathrm{B}) \\
\sim \\
\text { trapèze de Vielle }\end{array}$ \\
\hline $\begin{array}{l}\text { BG12 - géom. à } \\
\text { tronc. obliques par } \\
\text { ret. croisées }\end{array}$ & $\begin{array}{l}\text { bitronc. géo. dont } \\
\text { la plus grande } \\
\text { tronc. est façonnée } \\
\text { au moins en partie } \\
\text { par ret. croisées }\end{array}$ & & $\begin{array}{l}\text { BG12A - trapèze } \\
\text { BG12B - triangle }\end{array}$ & $\begin{array}{l}\text { BG12 } \\
(A, B)\end{array}$ \\
\hline $\begin{array}{l}\text { BG13 - géom. à } \\
\text { tronc. obliques et } \\
\text { ret. inv. ras. }\end{array}$ & $\begin{array}{l}\text { type BG11 ou } \\
\text { BG12 et retouches } \\
\text { inverses rasantes }\end{array}$ & & $\begin{array}{l}\text { BG13A - trapèze } \\
\text { BG13B - triangle }\end{array}$ & $\begin{array}{c}\text { BG13 } \\
(\mathrm{A}, \mathrm{B}) \\
\sim \\
\text { trapèze du Martinet } \\
\text { pointe de Sonchamp }\end{array}$ \\
\hline $\begin{array}{l}\text { BG14 - géom. à } \\
\text { tronc. obliques par } \\
\text { ret. croisées et ret. } \\
\text { dir. ras. }\end{array}$ & $\begin{array}{l}\text { type } \mathrm{BG} 12 \text { à } \\
\text { retouches directes } \\
\text { rasantes }\end{array}$ & & $\begin{array}{l}\text { BG14A - trapèze } \\
\text { BG14B - triangle }\end{array}$ & $\begin{array}{c}\text { BG14 } \\
(\mathrm{A}, \mathrm{B}) \\
\sim \\
\text { pointes de Gazel }\end{array}$ \\
\hline $\begin{array}{l}\text { BG15 - pointe } \\
\text { asym. à base } \\
\text { concave }\end{array}$ & $\begin{array}{l}\text { géo. à tronc. } \\
\text { oblique directe et } \\
\text { base concave } \\
\text { bifaciale }\end{array}$ & & $\begin{array}{l}\text { BG15A - étroite } \\
\text { BG15B - large }\end{array}$ & $\begin{array}{l}\sim \\
\text { armatures à éperon } \\
\text { pointes de Bavans }\end{array}$ \\
\hline $\begin{array}{l}\text { BG16 - pointe } \\
\text { asym. à base } \\
\text { concave et ret. ras. }\end{array}$ & $\begin{array}{l}\text { type BG15 à } \\
\text { retouches latérales } \\
\text { rasantes directes } \\
\text { ou inverses }\end{array}$ & & $\begin{array}{l}\text { BG16A - étroite } \\
\text { BG16B - large }\end{array}$ & pointes de Bavans \\
\hline $\begin{array}{l}\text { BG17 - autres } \\
\text { géom. à tronc. } \\
\text { obliques }\end{array}$ & $\begin{array}{l}\text { géo. à } 1 \text { ou } 2 \text { tronc. } \\
\text { obliques directes }\end{array}$ & & $\begin{array}{l}\text { BG17A - trapèze } \\
\text { BG17B - triangle }\end{array}$ & $\begin{array}{l}\text { BG15 } \\
(\mathrm{A}, \mathrm{B})\end{array}$ \\
\hline $\begin{array}{l}\text { BG21 - autre géom. } \\
\text { à bitronc. directes, } \\
\text { asym. }\end{array}$ & $\begin{array}{l}\text { trapèze à } 2 \text { tronc. } \\
\text { directes } \\
\text { d'obliquités } \\
\text { différentes }\end{array}$ & & $\begin{array}{l}\text { BG21A - long } \\
\text { BG21B - court }\end{array}$ & $\begin{array}{l}\mathrm{BG} 21 \\
(\mathrm{~A}, \mathrm{~B})\end{array}$ \\
\hline $\begin{array}{l}\text { BG22 - autre géom. } \\
\text { à bitronc. directes, } \\
\text { sym. }\end{array}$ & $\begin{array}{l}\text { géo. à } 2 \text { tronc. } \\
\text { directes de même } \\
\text { obliquité }\end{array}$ & & $\begin{array}{l}\text { BG22A - long } \\
\text { BG22B - court }\end{array}$ & $\begin{array}{l}\mathrm{BG} 22 \\
(\mathrm{~A}, \mathrm{~B})\end{array}$ \\
\hline
\end{tabular}

Fig. 2 - Structuration typologique des types 1 et 2 au sein de la classe des bitroncatures géométriques.

chaque site. Les autres gisements stratifiés de l'arc jurassien attribués au Mésolithique ont alors été écartés car leurs industries sont insuffisamment décrites dans les publications consultées (absence de décomptes détaillés et/ou de dessins).

\section{ANALYSE}

Le tableau de données a été traité par analyse factorielle des correspondances (AFC). Les couches à faibles effectifs (inférieurs à une trentaine d'individus) ou dont la cohérence stratigraphique est contredite par les résultats de l'analyse, ont été passées en individus supplémentaires (à l'exception toutefois de la couche $4 \mathrm{~b}$ de l'abri Freymond). Les résultats de l'AFC sont illustrés par la figure 4 .

La structure obtenue sur les deux premiers axes factoriels rassemble $79 \%$ de la signification. Elle se caractérise par un double effet Guttman sur les individus et les critères. Le premier axe factoriel, qui exprime $61 \%$ 
Fig. 3 - Carte de répartition des principaux sites du Mésolithique récent et final de l'arc jurassien et de ses marges.

008 : abri du Roseau (Neuville-sur-Ain, 01);

009 : grotte du Souhait (Montagnieu, 01);

010 : Sous-Balme (Culoz, 01);

011 : abri de Sous-Sac (Injoux-Génissiat, 01)

012 : Sous-Vargonne (Andert-Candon, 01);

013 : la Touvière (Arbignieu, 01);

037 : Bavans (25);

040 : baume de Montandon (Saint-Hippolyte, 25);

042 : abri de la Roche-aux-Pêcheurs (Villers-le-Lac, 25);

051 : Bouvante (38);

076 : Aulp-du-Seuil, abris n ${ }^{\text {os }} 1$ et 3 (Saint-Bernard-du-Touvet, 38)

083 : Coufin I et II (Choranche, 38);

085 : la Grande Rivoire (Sassenage, 38);

086 : Pas de la Charmatte (Chatelus, 38);

087 : abri des Cabônes (Ranchot, 39);

092 : À Daupharde (Ruffey-sur-Seille, 39);

112 : Mannlefelsen I (Oberlarg, 68);

119 : les Mézières (Mantoche, 70);

144 : Birsmatten (Nenzlingen, BE);

146 : Liesbergmühle VI (Liesberg, BE);

147 : Ritzigrund (Roggenburg, BE);

148 : Tschäpperfels (Röschenz, BE);

150 : les Gripons (Saint-Ursanne, JU);

151 : Schötz 7 (Schötz, LU);

153 : le Col des Roches (Le Locle, NE);

156 : Château d'Oex (VD);

157 : abri de la Cure (Baulmes, VD);

158 : abri Freymond (Mont-la-Ville, VD);

167 : abri Vionnaz (Collombey Muraz, VS);

178 : abri Gay (Poncin, Ain);

180 : abri du Sault (Serrières-sur-Ain, 01);

201 : abri Gigot (Brétonvillers, 25);

222 : grotte de la Margot (Montrond, 39).

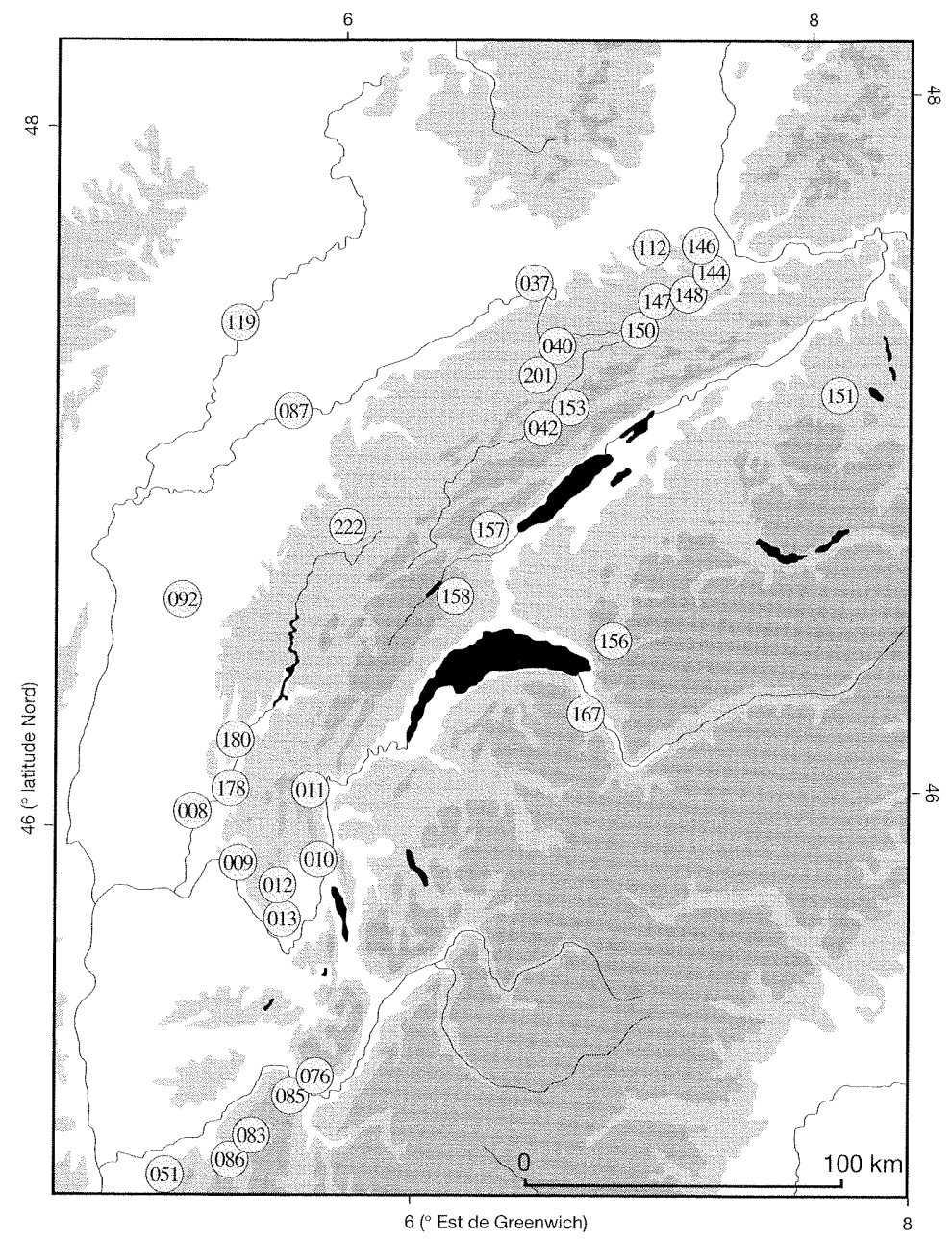

de la structuration, a valeur chronologique. Il est principalement construit par une opposition entre les lamelles étroites à bord abattu (BA11 et BA12) et les lames à enlèvements irréguliers (IR22), qui se traduit par une opposition entre la couche 7 de l'abri Central de Bavans d'une part, et Schötz 7 de l'autre. L'axe 2, avec $18 \%$ de la structure, oppose les lamelles à bord abattu (BA11) aux géométriques à troncature oblique (BG1). Avec $10 \%$ de la structure, 1'axe 3 se caractérise lui par l'opposition entre les lames à bord abattu (BA23) et les géométriques à troncature oblique (BG11-13). Il est également surprenant de constater que les pointes asymétriques à base concave (BG15-16) ne s'expriment fortement que sur l'axe 6, lequel ne représente que $1,2 \%$ de la structure générale ! Notons quand même que cet axe se construit par une opposition entre ces pointes asymétriques et les géométriques à bitroncature directe symétriques (BG22).

L'analyse factorielle prend également en compte 19 individus supplémentaires qui viennent se placer de façon plus ou moins cohérente sur la structure. Le cas de l'abri des Gripons c. 4 (Mésolithique moyen) est particulièrement intéressant. Si on le laisse en effet dans la structure principale, il écrase l'ensemble par une opposition extrêmement marquée entre lui et l'ensemble des autres gisements. Cet effet est dû à une surreprésentation des armatures de flèches (triangles scalènes) dans cette industrie. Contrairement à ce que concluaient les auteurs (Pousaz et al., 1991, p. 146), cela nous conduit à penser que la constitution de l'industrie de la couche 4 est bien liée à une fonction préférentielle, la chasse. Si d'autres activités sont évidemment présentes (débitage, grattage de peaux et de fourrures, etc.; ibid.), elles peuvent d'ailleurs toutes être comprises comme des activités annexes des pratiques cygénétiques.

Si le double effet Guttman obtenu permet d'interpréter les résultats de cette AFC comme une sériation typochronologique, il apparaît nettement que celle-ci n'est pas continue, qu'il existe des ruptures. La réalisation d'une classification ascendante hiérarchique $(\mathrm{CAH})$ sur les coordonnées des individus sur les deux premiers axes factoriels permet de bien mettre en évidence cet effet de partition (fig. 5). Cette CAH montre l'existence de plusieurs groupes, certains se subdivisant nettement en deux (mais sous le seuil statistique). Ces groupes peuvent être interprétés en termes de typochronologie. On individualise ainsi nettement le Mésolithique ancien (et l'Épipaléolithique terminal). La structure du Mésolithique moyen est plus complexe. Il semble en effet que l'on puisse y distinguer deux groupes : le premier rassemblerait les horizons 3 à 5 de Birsmatten, 


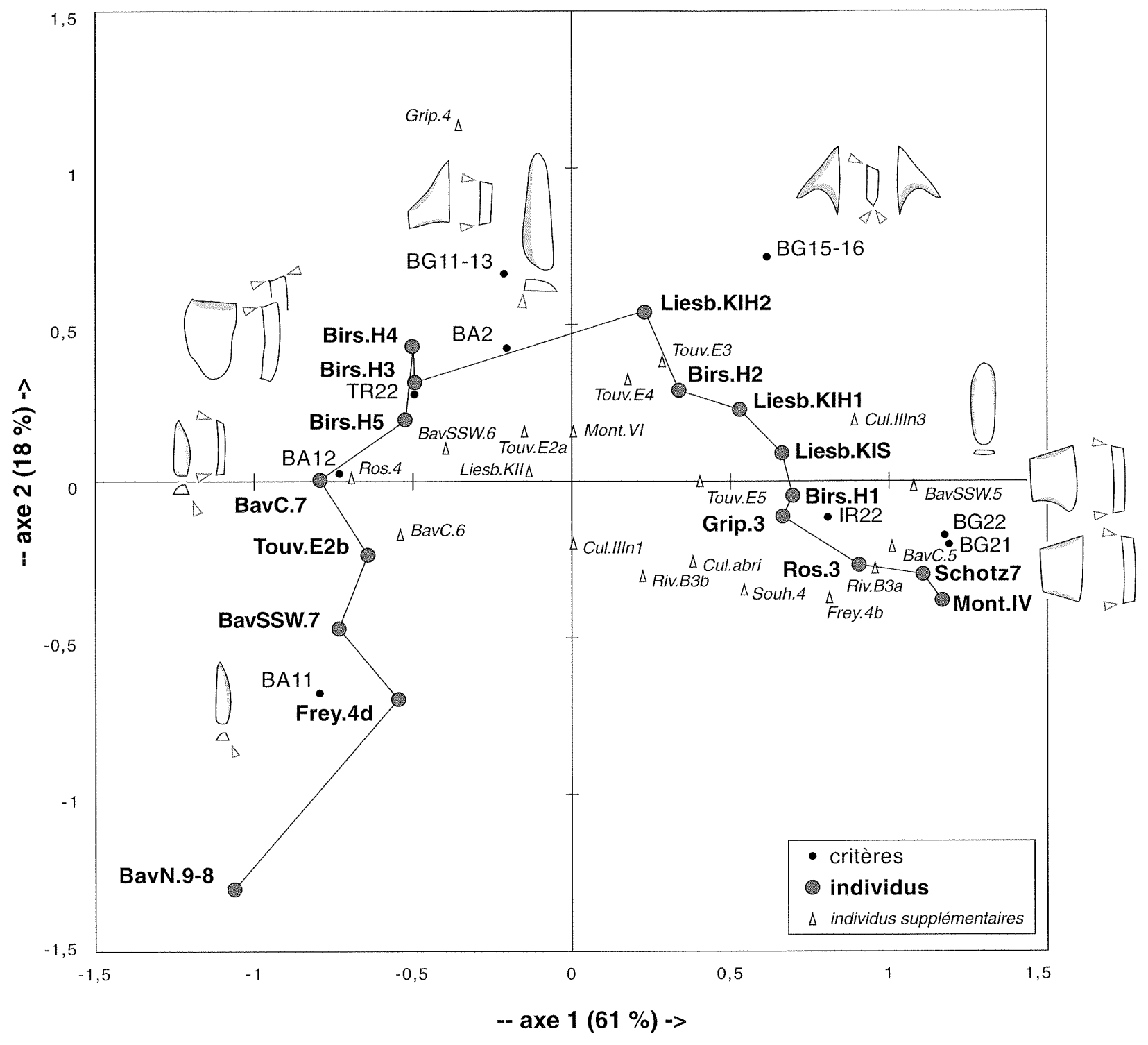

Individus : BavN.9-8 : grotte Nord de Bavans, c. 9 et 8 ; Frey.4d : abri Freymond, c.4d ; BavSSW.7 : abri SudSud-Ouest de Bavans, c.7 ; Touv.E2b : abri de la Touvière, E2b ; BavC.7 : abri Central de Bavans, c.7 ; Birs.H5 : Birsmatten Basisgrotte, Horizont 5 ; Birs.H4 : Birsmatten Basisgrotte, Horizont 4 ; Birs.H3 : Birsmatten Basisgrotte, Horizont 3 ; Liesb.KIH2 : Liesbergmühle VI, Komplex I, Horizont 2 ; Birs.H2 : Birsmatten Basisgrotte, Horizont 2 ; Liesb.KIH1 : Liesbergmühle VI, Komplex I, Horizont 1 ; Liesb.KIS : Liesbergmühle VI, Komplex I, Sondierung ; Birs.H1 : Birsmatten Basisgrotte, Horizont 1 ; Grip.3 : abri des Gripons, c.3 ; Ros.3 : abri du Roseau, c.3 ; Schötz7 : Schötz 7 ; Mont.IV : abri de Montandon, c.IV.

Individus supplémentaires : BavC.6 : abri Central de Bavans, c. 6 ; BavSSW.6 : abri Sud-Sud-Ouest de Bavans, c.6 ; Ros. 4 : abri du Roseau, c.4 ; Liesb.KII : Liesbergmühle VI, Komplex II ; Grip.4 : abri des Gripons, c.4 ; Touv.E2a : abri de la Touvière, E2a ; Mont.VI : abri de Montandon, c.VI ; Touv.E5 : abri de la Touvière, E5 ; Cul.IIIn3 : Culoz, couhe III niveau 3 ; BavSSW.5 : abri Sud-Sud-Ouest de Bavans, c. 5 ; Cul.IIIn1 : Culoz, couche III niveau 1 ; Cul.abri : Culoz, abri ; Riv.B3b : abri de la Grande Rivoire, c.B3b ; Souh.4 : grotte de Souhait, c.4 ; Frey. $4 b$ : abri Freymond, c.4b ; BavC.5 : abri Central de Bavans, c.5 ; Riv.B3a : abri de la Grande Rivoire, c.B3a ; Touv.E3 : abri de la Touvière, E3 ; Touv.E4 : abri de la Touvière, E4.

Critères : BA11 : lamelles étroites à bord(s) abattu(s) aiguë ; BA12 : lamelles étroites à bord(s) abattu(s) aiguë, tronquée ; TR22 : troncatures normales; BA2 : lames à bord abattu ; BG11-13 : géométriques à troncature oblique ; BG15-16 : pointes asymétriques à base concave ; IR22 : lame(lle)s à enlèvements irréguliers ; BG22 : géométriques à bitroncatures directes symétriques ; BG21 : géométriques à bitroncatures directes asymétriques.

Fig. 4 - Résultat graphique de l'analyse factorielle des correspondances : profils lignes et profils colonnes sur les axes 1 et 2. 

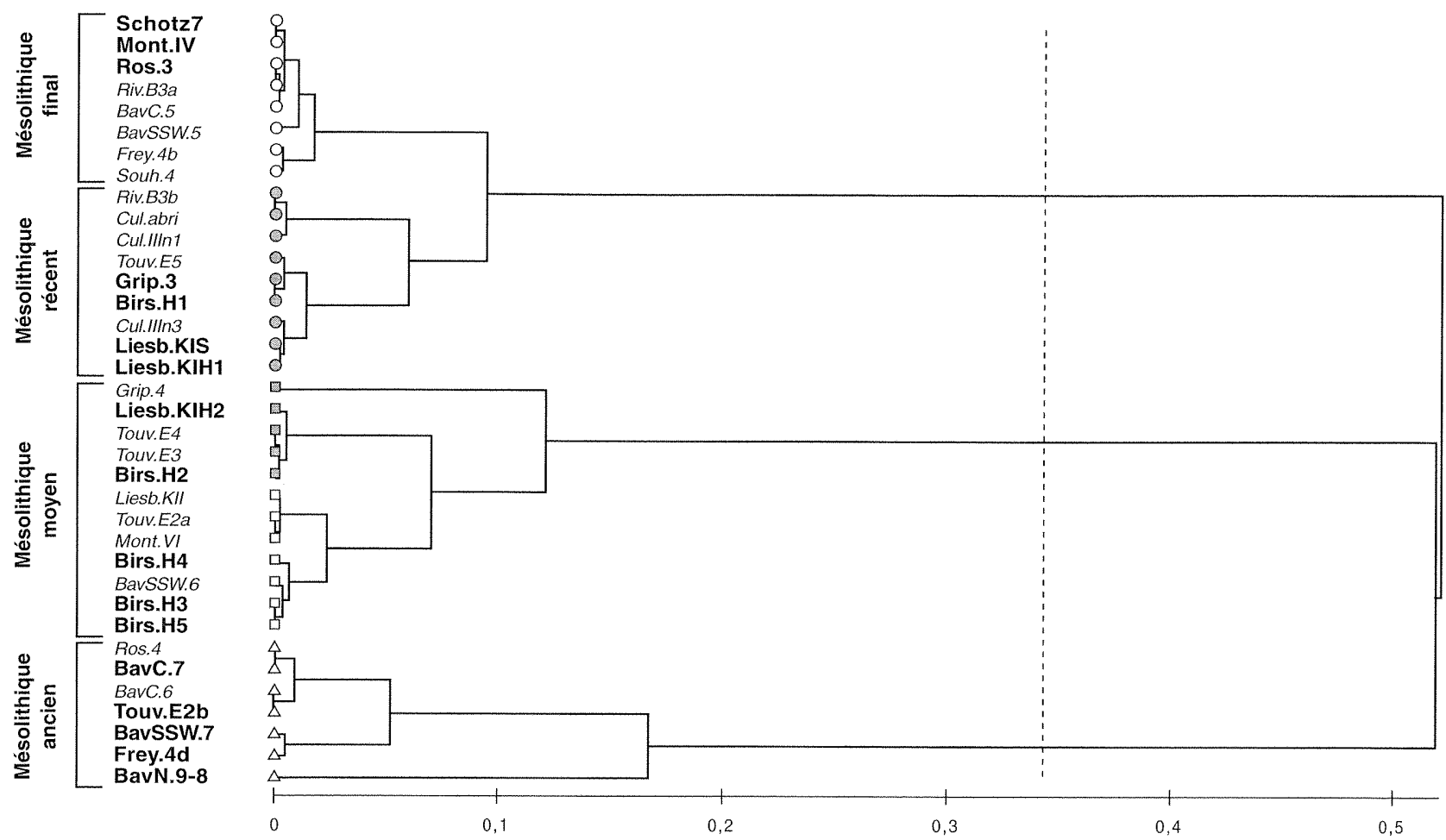

Fig. 5 - Classification ascendante hiérarchique sur les coordonnées des individus sur les deux premiers axes factoriels.

et le second comprendrait Birsmatten $\mathrm{H} 2$ et Liesbergmühle KIH2.

Pour les périodes récentes du Mésolithique, qui nous intéressent le plus ici, on distingue :

- le Mésolithique récent, caractérisé par l'association lamelles à enlèvements irréguliers (IR22), trapèzes asymétriques (BG21) et armatures asymétriques à base concave (BG15), qui ne seraient alors plus des éléments caractéristiques du Mésolithique final régional, nous y reviendrons;

- et le Mésolithique final, caractérisé par un assemblage peu ou prou semblable mais où dominent les trapèzes (BG21 et BG22), en rupture morphologique par rapport aux formes antérieures.

Avant de pouvoir proposer une interprétation chronoculturelle de ces résultats, notamment en ce qui concerne la fin de cette période, il est d'abord indispensable de confronter la structuration obtenue aux données de la chronologie absolue, de manière à fixer dans le temps les diverses étapes individualisées.

\section{PERSPECTIVES CHRONOLOGIQUES}

Le Mésolithique récent et final de l'arc jurassien a fait l'objet d'une série de dates importantes. Malheureusement, beaucoup d'entre elles doivent être écartées, soit par manque de fiabilité physico-chimique, soit par incohérence, soit par manque de lien archéologique (fig. 6 et 7). C'est ainsi le cas pour la couche 5 des abris de Bavans où, au vu des problèmes stratigraphiques et de l'incohérence des dates, nous rejetons l'ensemble de la série, bien que Lv 1415 datant la base de la couche 5 entre 6070-5890 av. J.-C. puisse se rapporter au Mésolithique récent/final. De même, les dates de Birsmatten $\mathrm{H} 1$ et $\mathrm{H} 2$ sont trop contradictoires entre elles pour pouvoir être retenues. La date Gif 8397 de l'abri des Cabônes n'est, dans la littérature (Cupillard, 1998a), pas clairement associée au Mésolithique récent et doit donc, pour l'instant, être écartée. L'ensemble des dates de la couche 3 de l'abri des Gripons doit être rejeté en raison de son incohérence. La date de Liesbergmühle VI doit être écartée par manque de fiabilité du contexte. Les deux dates de la c. IV de la baume de Montandon sont légèrement incohérentes entre elles. En attendant de nouveaux résultats $(\mathrm{Cu}-$ pillard et al., 2000), nous préférons les écarter temporairement. Enfin, avec des écarts types trop importants, les dates de Culoz et de l'abri de la Touvière doivent également être rejetées.

En définitive, pour le Mésolithique récent et final de cette région, nous ne pouvons retenir que trois dates sur plus d'une quinzaine. De plus, il apparaît que ces trois dates se rapportent toutes à notre Mésolithique final. Ceci implique que le Mésolithique récent de l'arc jurassien ne dispose actuellement d'aucune date fiable. La réalisation d'un histogramme cumulatif pondéré (Sabatier, 1997), malgré le faible nombre de dates mobilisables, permet cependant d'aller au-delà de ce constat (fig. 8). Il montre en effet que le stade final (d'après les industries) du Mésolithique se situerait entre 6200 et 5700 av. J.-C. (ce résultat confirme celui de Voruz, 1999). Nous avons également placé sur cette figure l'histogramme du Mésolithique moyen 


\begin{tabular}{|c|c|c|c|c|c|}
\hline Référence & Site & Couche & Matériel daté & Mesure (en BP) & Calibration (à 1 o) \\
\hline \multicolumn{6}{|c|}{ Dates retenues } \\
\hline LV 1455 & Bavans Sud-Sud-Ouest (25) & c. 6 & os & $8210 \pm 80$ & $7450-7080$ \\
\hline UZ 2478 & Gripons (JU) & c. 4 & charbons & $8405 \pm 90$ & $7540-7310$ \\
\hline UZ 2477 & Gripons (JU) & c.4 foyer & charbons & $8660 \pm 100$ & $7890-7540$ \\
\hline UZ 2598 & Gripons (JU) & c.4 foyer & charbons & $8850 \pm 85$ & $8010-7710$ \\
\hline UZ 2370 & Gripons (JU) & c. 4 fosse & charbons & $8960 \pm 125$ & $8100-7730$ \\
\hline Gif 6967 & Montandon (25) & c.VI & charbons & $8490 \pm 110$ & $7650-7350$ \\
\hline \multicolumn{6}{|c|}{ Dates rejetées } \\
\hline B 235 & Birsmatten (BE) & horizon 2 & os & $5310 \pm 240$ & $4450-3800$ \\
\hline B 240 & Birsmatten (BE) & horizon 2 & os brûlé & $7200 \pm 600$ & $6800-5400$ \\
\hline B 241 & Birsmatten (BE) & $\mathrm{H} .3$ & os brûlé & $7480 \pm 200$ & $6500-6080$ \\
\hline B 236 & Birsmatten (BE) & $\mathrm{H} .3$ & os & $6970 \pm 120$ & $5980-5730$ \\
\hline OXA 2238 & Birsmatten (BE) & H.3 (sépulture) & os & $6290 \pm 80$ & $5370-5080$ \\
\hline B 237 & Birsmatten (BE) & $\mathrm{H} .4$ & os & $7670 \pm 120$ & $6650-6400$ \\
\hline B 238 & Birsmatten (BE) & H.5 & os & $7460 \pm 160$ & $6460-6100$ \\
\hline UZ 2597 & Gripons (JU) & c.4 sup. & charbons & $6540 \pm 80$ & $5570-5340$ \\
\hline UZ 2599 & Gripons (JU) & c. 4 fosse & charbons & $7205 \pm 80$ & $6120-5950$ \\
\hline Ly 619 & Touvière (01) & E4 & charbons & $6850 \pm 420$ & $6200-5300$ \\
\hline
\end{tabular}

Fig. 6 - Tableau des dates disponibles pour le Mésolithique moyen de l'arc jurassien (au sein des sites pris en compte dans l'analyse statistique).

\begin{tabular}{|c|c|c|c|c|c|}
\hline Référence & Site & Couche & Matériel daté & Mesure (en BP) & Calibration (à 1 o) \\
\hline \multicolumn{6}{|c|}{ Dates retenues } \\
\hline CRG 579 & abri Freymond (VD) & c. $4 b$ & charbons & $7190 \pm 140$ & $6220-5910$ \\
\hline B 726 & Schötz $7($ LU) & - & $?$ & $7080 \pm 130$ & $6070-5800$ \\
\hline B 728 & Schötz 7 (LU) & - & $?$ & $6980 \pm 90$ & $6020-5710$ \\
\hline \multicolumn{6}{|c|}{ Dates rejetées } \\
\hline Gif 6058 & abri de Bavans (25) & c.5 niv. ind. & os & $4310 \pm 90$ & $3100-2700$ \\
\hline Gif 5165 & abri de Bavans (25) & c. 5 niv. 1 (sommet) & os & $5320 \pm 120$ & $4320-3990$ \\
\hline Lv 1588 & abri de Bavans (25) & c. 5 niv. 3 (milieu) & os & $6500 \pm 100$ & $5610-5360$ \\
\hline Lv 1590 & abri de Bavans (25) & c. 5 niv. 3 (milieu) & $?$ & $6410 \pm 95$ & $5480-5310$ \\
\hline Lv 1415 & abri de Bavans (25) & c. 5 niv. 5 (base) & os & $7130 \pm 70$ & $6070-5890$ \\
\hline B 234 & Birsmatten (BE) & horizon 1 & os & $5350 \pm 120$ & $4330-4040$ \\
\hline Ly 289 & Culoz (01) & c. III niv.1 & charbons & $7360 \pm 1080$ & $7600-5100$ \\
\hline Ly 287 & Culoz (01) & c. 111 niv. 3 & charbons & $4790 \pm 140$ & $3710-3370$ \\
\hline Ly 288 & Culoz (01) & c.III niv. 3 & charbons & $4600 \pm 480$ & $4000-2600$ \\
\hline UZ 2371 & abri des Gripons (JU) & c. 3 & charbons & $6510 \pm 110$ & $5560-5320$ \\
\hline UZ 2479 & abri des Gripons (JU) & c. 3 & charbons & $5965 \pm 80$ & $4940-4770$ \\
\hline UZ 2596 & abri des Gripons (JU) & c. 3 & charbons & $4195 \pm 70$ & $2890-2620$ \\
\hline B 2161 & Liesbergmühle VI (BE) & Komplex I & charbons & $6220 \pm 340$ & $5450-4700$ \\
\hline Gif 6966 & baume de Montandon (25) & c. IV & charbons & $6038 \pm 80$ & $5040-4800$ \\
\hline Gif 6608 & baume de Montandon (25) & c. IV & charbons & $5890 \pm 100$ & $4910-4610$ \\
\hline Ly 5923 & abri du Roseau (01) & c. 3 & os épars & $6140 \pm 70$ & $5210-4950$ \\
\hline GrA 16351 & abri du Roseau (01) & c. 3 & $?$ & $6720 \pm 40$ & $5700-5560$ \\
\hline Ly 269 & abri de la Touvière $(01)$ & c.s.m.o. $(=E 5)$ & charbons & $6190 \pm 160$ & $5310-4860$ \\
\hline
\end{tabular}

Fig. 7 - Tableau des dates disponibles pour le Mésolithique récent et final de l'arc jurassien (au sein des sites pris en compte dans l'analyse statistique).

jurassien, réalisé à partir de sept dates provenant de la c. 4 de l'abri des Gripons, de la couche 6 de Bavans et de la c. VI de la baume de Montandon. Ce Mésolithique moyen viendrait alors se placer entre 8000 et 7100 av. J.-C.

Remarquons ici que cet histogramme montre l'existence de deux pics de probabilités. Malgré le faible nombre de datations mobilisables, on ne peut manquer de mettre en relation cette bimodalité de l'histogramme avec la bipartition chronotypologique que suggérait l'AFC et la CAH. La fourchette chronologique comprise entre 7100 et 6200 pourrait correspondre au Mésolithique récent jurassien de type Birsmatten $\mathrm{H} 2-\mathrm{H} 1$. Il serait alors contemporain du Mésolithique récent des Alpes du nord daté entre 7100 et 6500 av. J.-C. (Perrin, 2001). 


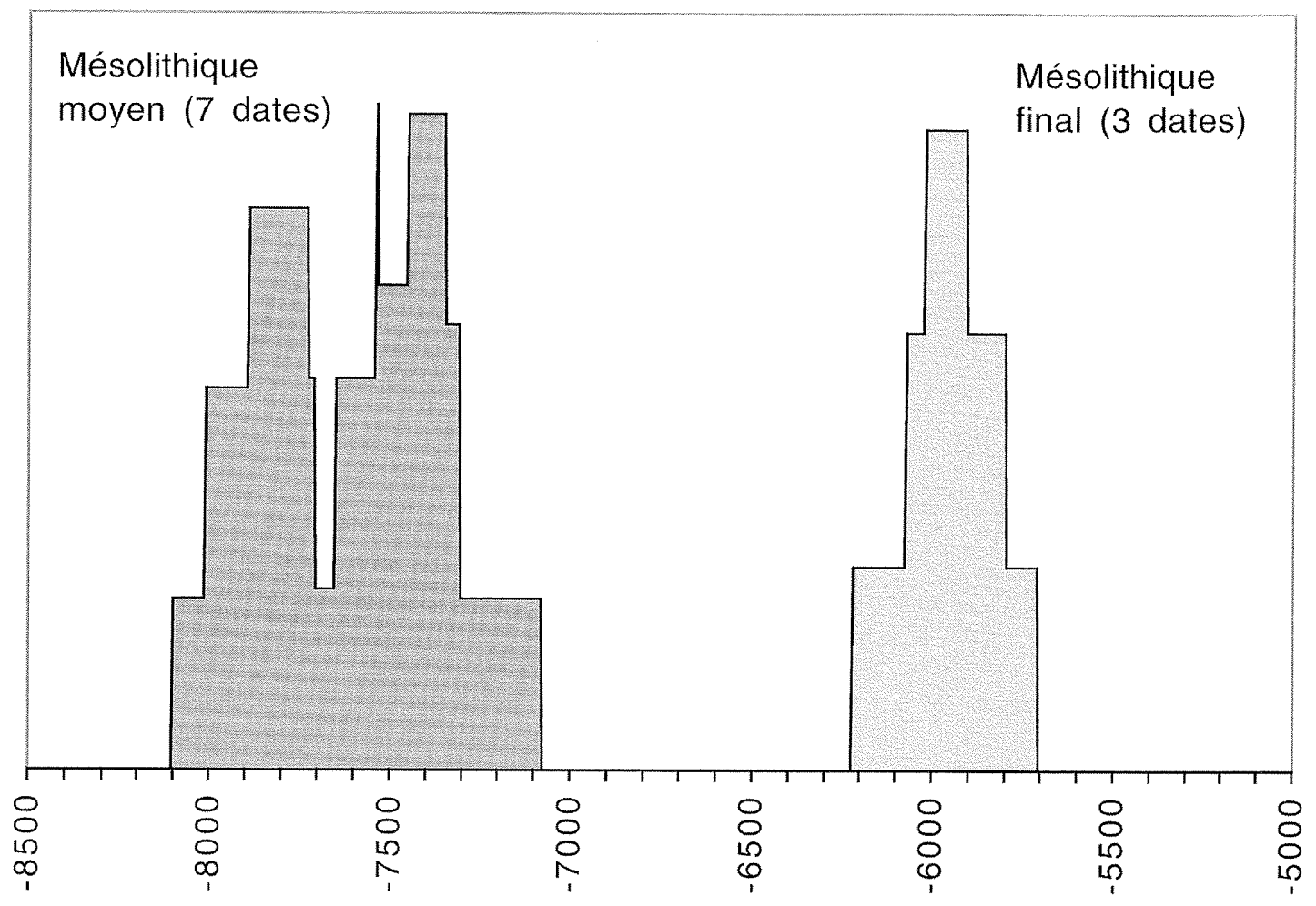

Fig. 8 - Histogramme cumulatif pondéré des dates retenues pour le Mésolithique moyen à final de l'arc jurassien.

\section{LA QUESTION DES ENSEMBLES MIXTES}

Ainsi, il apparaît clairement que, malgré la déficience importante de dates fiables, la structuration chronotypologique des industries mésolithiques de l'arc jurassien que nous avons obtenue trouve un écho cohérent dans les données chronologiques régionales. Cependant, si l'on s'en tient strictement aux datations retenues, force est de constater l'existence d'une rupture chronologique marquée entre ce Mésolithique final et le premier Néolithique ancien, attesté régionalement à partir de 5200 av. J.-C. environ (Voruz, 1999; Perrin, 2001). Dès lors, comment, par exemple, expliquer la présence d'armatures perçantes asymétriques à base concave bifaciale (BG15) à la grotte du Gardon dans des couches datées entre 5200 et 4900 av. J.-C. (Perrin, 2001)? De même, au sein des dates rejetées, le nombre de mesures venant précisément se placer au sein de seconde moitié du $\mathrm{VI}^{\mathrm{e}}$ millénaire reste troublant.

L'hypothèse optimiste consiste à identifier, sous ce faisceau de contradictions, les traces de l'existence "d'ensembles mixtes" (Manen, 1997; Voruz, 1999). Ceux-ci seraient l'expression de l'acculturation vers le Néolithique de populations mésolithiques. Cette acculturation se traduirait alors par la présence d'animaux domestiques au sein d'ensembles que l'industrie lithique attribue au Mésolithique, et/ou par la présence de traits matériels néolithiques (notamment la céramique) au sein d'assemblages toujours à industrie mésolithique (fig. 9). Si cette hypothèse est extrêmement séduisante, il n'en reste pas moins qu'elle repose sur des bases extrêmement fragiles. Du point de vue du lithicien en tout cas, lorsque sont attestées des pièces néolithiques au sein d'ensembles mésolithiques, ou inversement, il n'est jamais possible d'exclure l'hypothèse d'intrusions liées à des contextes sédimentaires complexes (comme la

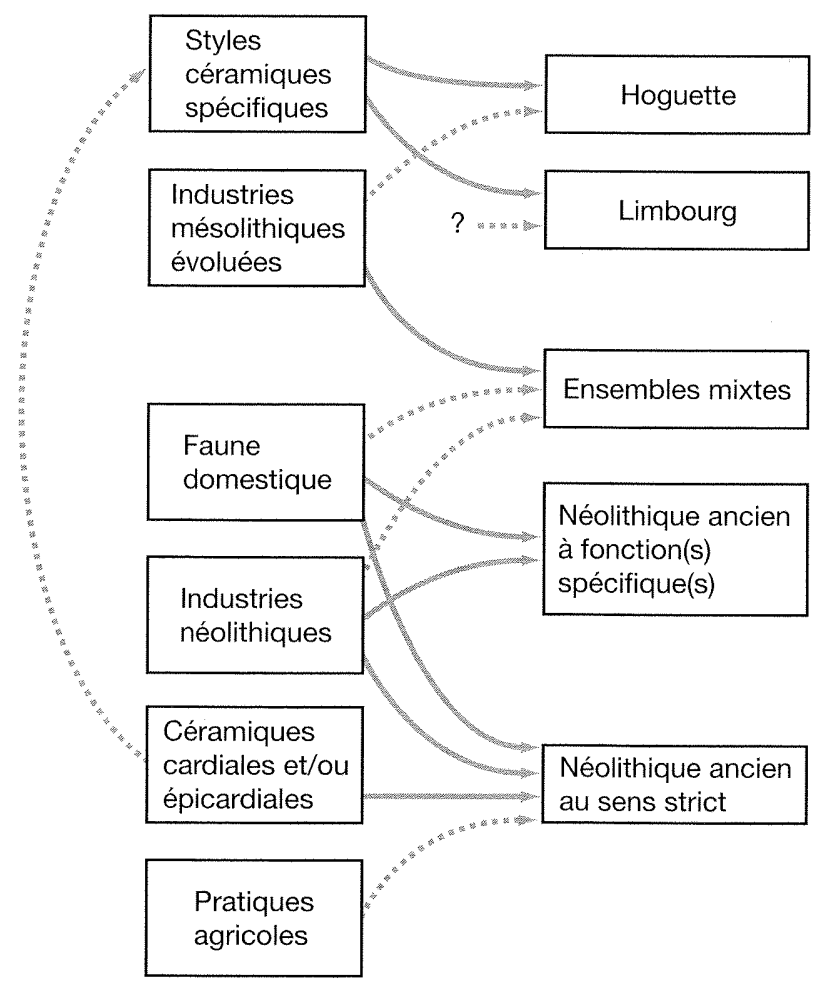

Fig. 9 - L'expression de la néolithisation du Bassin rhodanien : schéma conceptuel des diverses possibilités. 
couche 5 de Bavans par exemple). Régionalement, les sites de Culoz (Vilain, 1966) et de la Touvière (Morelon, 1973) présenteraient ainsi des industries "mixtes" entre le Mésolithique final et le Néolithique ancien. Malheureusement, à l'abri de la Touvière par exemple, si plusieurs géométriques à bitroncatures inverses caractéristiques du Néolithique ancien méridional sont effectivement attestés, ceux-ci coexistent avec plusieurs tessons décorés au peigne, indubitablement campaniformes (conservés au Département de Géologie de 1'Université de Lyon I)...

\section{LA QUESTION \\ DES STYLES CÉRAMIQUES SPÉCIFIQUES}

Les exemples les plus connus de ces ensembles mixtes seraient illustrés par les styles céramiques de la Hoguette et du Limbourg. Si ces deux styles céramiques sont souvent mobilisés dans les discussions sur les processus de néolithisation, l'interprétation de leur genèse reste controversée : Mésolithique acculturé ou en voie de néolithisation pour les uns, Néolithique à part entière pour les autres. Nous n'avons pas ici la prétention de vouloir trancher en faveur de l'une ou l'autre des hypothèses, mais nous voulons vérifier la pertinence de cette affirmation, certes attrayante, qui voudrait que "l'industrie lithique associée à la céramique de la Hoguette est d'empreinte mésolithique" (Mazurié de Keroualin, 2000 , p. 366). Cet argument est d'importance, puisqu'il mène certains auteurs à ne plus simplement parler de style céramique de la Hoguette, mais de culture à part entière (Schütz et al., 1991).

Pour pouvoir discuter de l'industrie associée à ce style céramique, il faut revenir aux contextes de découvertes de ces tessons lorsqu'ils sont supposés être associés à des industries mésolithiques. Dans le tableau de la fig. 10, nous présentons l'ensemble des sites ayant livré de la céramique du style de la Hoguette et n'étant pas des habitats rubanés (cet inventaire est réalisé d'après le catalogue de sites de Manen, 1997). Sur 49 sites recensés, seuls 10 peuvent entrer dans cette discussion d'une attribution ou non au Mésolithique récent/final. L'examen des contextes de découvertes réduit ce nombre à 3 : Oberlarg Mannlefelsen c. G, Stuttgart Bad-Cannstatt et Inzigkofen-Vilsingen Dietfurt.

En ce qui concerne le niveau G de l'abri du Mannlefelsen I à Oberlarg, C. Jeunesse (1986) signale un tesson décoré au peigne typique de la céramique de la Hoguette. Selon lui, d'autres exemplaires non décorés proviendraient également de ce niveau. Mais A. Thévenin et J. Sainty ne font jamais allusion à de tels éléments, même dans leurs articles les plus récents (Sainty et Thévenin, 1998). Quoi qu'il en soit, l'industrie lithique taillée du niveau $G$ n'est pas connue. On sait simplement que les niveaux $\mathrm{G}$ à $\mathrm{L}$ dans leur ensemble correspondent à un "Mésolithique de transition sur débitage régulier, avec lamelles à bords parallèles, à nervures régulières et épaisseur constante entre 2 et $3 \mathrm{~mm}$ " (Thévenin et Sainty, 1974). En attendant une nouvelle étude de cette série, il n'est donc pas possible de retenir ce site comme preuve de la coexistence d'une industrie mésolithique et de tessons du style de la Hoguette.

Le site de Dietfurt à Inzigkofen-Vilsingen a livré lui une céramique non décorée, attribuée au style de la Hoguette sur la base de sa proximité stylistique avec les éléments de Stuttgart Bad-Cannstatt (Gelhen, 1992). Mais la stratigraphie de ce gisement ne permet pas d'individualiser de niveaux archéologiques nets. Si les auteurs reconnaissent la présence d'une industrie du Mésolithique récent (ibid.), il n'est donc pas possible de la corréler aux tessons du style de La Hoguette.

Enfin, le site de Stuttgart Bad-Cannstatt est celui qui est le plus mobilisé dans cette discussion. Les fouilleurs y auraient en effet identifié de la céramique du style de la Hoguette, associée à des ovicaprinés, à des lames régulières et à des harpons (Schütz et al., 1991). Si la couche archéologique paraît bien être cohérente, il faut cependant souligner que ce site n'est connu que par l'intermédiaire de deux sondages de $2 \mathrm{~m}^{2}$ chacun, en sus du rare matériel issu des premières fouilles. Ainsi, l'assemblage ne comprend au total (sondages et fouilles anciennes) que quelques tessons (une dizaine?) dont certains décorés et/ou dégraissés à l'os, une centaine d'ossements et deux outils en silex (1 lame à retouches directes et 1 armature microlithique, qui n'est par ailleurs pas décrite). En définitive, l'attribution objective de cet ensemble au Mésolithique ne tient que par la présence de fragments de harpons en bois de cerf. Ainsi, l'association céramique décorée du style de la Hoguette et industrie lithique de tradition mésolithique ne peut en aucun cas être objectivement démontrée de manière directe, pas plus semble-t-il d'ailleurs que pour la céramique du Limbourg (van Berg, 1991). Quelques indices laissent cependant penser que l'hypothèse de chasseurs recevant voire fabriquant de la céramique pourrait effectivement être la bonne : origine

\begin{tabular}{|l|l|l|}
\hline \multicolumn{1}{|c|}{ Site } & Couche & \multicolumn{1}{|c|}{ Contexte } \\
\hline Brétonvilliers, abri de Gigot I & & hors contexte \\
\hline Le Locle, Col des Roches & & hors contexte \\
\hline Neuville-sur-Ain, abri du Roseau & & hors contexte \\
\hline Baulmes, abri de la Cure & fosse & entre Mésolithique "supérieur" et Vallon-des-Vaux \\
\hline Bavans & c.5 & mélanges (Mésolithique récent / Rubané récent) \\
\hline \hline Oberlarg Mannlefelsen & c.G & Mésolithique tardif \\
\hline Inzigkofen-Vilsingen, Dietfurt & & Mésolithique récent \\
\hline Stuttgart, Bad Cannstatt & & Mésolithique récent / final \\
\hline
\end{tabular}

Fig. 10 - Lise des sites ayant livré des tessons du style de la Hoguette hors contexte rubané (d'après Manen, 1997). 
méditerranéenne des concepts stylistiques des décors céramiques (Jeunesse, 1987; Manen, 1997), preuve de la fabrication sur place, à Bavans, de la céramique de la Hoguette alors que la céramique rubanée est importée (Aimé, 1993). Cependant, seule la découverte de contextes stratigraphiques fiables et bien documentés pourra renouveler la recherche dans cette question. Cette critique des contextes de découvertes ne veut pas dire que nous réfutons l'existence des ensembles mixtes, ils ont nécessairement existé, ou même celle d'une possible "culture Hoguette", mais en l'état des données, cette dernière affirmation relève plus de la croyance que de l'analyse objective de faits archéologiques.

\section{CONCLUSION}

Nous espérons avoir montré, dans cet article, qu'une approche statistique des industries lithiques taillées, si elle ne constitue pas une démarche particulièrement novatrice (cf. par exemple Rozoy, 1978; Decormeille et Hinout, 1982 ; Hinout, 1984; etc.), peut cependant s'avérer riche d'enseignements et de perspectives. Il est ainsi possible de structurer entre eux l'ensemble des sites mésolithiques jurassiens. Si nous nous sommes ici cantonné aux gisements stratifiés (à l'exception de Schötz 7), il serait certainement utile d'y adjoindre les sites de plein air. Ce travail, qui reste à faire, permettrait de gommer une possible particularité fonctionnelle des gisements.

Quoi qu'il en soit, ces analyses nous permettent de dégager plusieurs points d'importance :

- la possible partition du Mésolithique moyen en deux phases, tant d'un point de vue chronologique que typologique;

- la mise en évidence nette des phases récente et finale du Mésolithique jurassien. Leur partition repose sur des données surprenantes par rapport aux schémas "traditionnels" qui considèrent en effet que le Mésolithique final se caractérise par l'apparition des armatures évoluées, comme les pointes de Bavans. Ici, nous voyons que ces armatures perçantes asymétriques à base concave bifaciale (BG15 et 16) apparaissent dès le Mésolithique récent, soit entre 7100 et 6200 av. J.-C. À Birsmatten par exemple, ces pièces existent dès l'horizon 2 (Rozoy, 1978). Elles sont également présentes dans l'horizon $1 \mathrm{du}$ Komplex I de Liesbergmühle (Hofmann-Wyss, 1978). Cette conjonction typologique montre bien que l'armature asymétrique à base concave reste conceptuellement dans la ligne évolutive mésolithique. Présentes dès le Mésolithique récent, ces pièces continuent bien sûr à exister au Mésolithique final, et même chronologiquement jusqu'au Néolithique ancien (par exemple à la grotte du Gardon : Perrin, 2001). Mais ce Mésolithique final, daté entre 6200 et 5700 av. J.-C., s'individualise principalement par la présence de géométriques à bitroncatures directes (qui coexistent avec les armatures asymétriques).

Dans la zone qui nous concerne, ces trapèzes sont en rupture complète avec les formes antérieures. II n'existe notamment pas de situation similaire à celle de Montclus 15-16 où les trapèzes apparaissent fortement dans des niveaux attribués à la fin du Mésolithique moyen ("Montclusien récent" : Rozoy, 1978). Ces deux couches de Montclus ne sont datées que par une unique mesure entre 6560 et 6220 av. J.-C. (Ly $542: 7540 \pm 160 \mathrm{BP}$ - date sur os du niveau 16 ), ce qui les situerait de façon légèrement antérieure à ce que nous avons dénommé ici "Mésolithique final jurassien”. Montclus 15-16 témoignerait ainsi d'une apparition précoce des industries à lames et trapèzes dans le sillon rhodanien. L'apparition plus tardive dans le Jura de ces industries pourrait donc résulter d'une évolution formelle sous l'influence de ces groupes mésolithiques déjà en possession de trapèzes.

Mais ces trapèzes pourraient également être le reflet d'un emprunt aux populations du Néolithique ancien méditerranéen. Cet emprunt se limiterait aux aspects formels des armatures (convergence des formes) sans prise en compte des aspects technologiques (bitroncature directe pour le Mésolithique, inverse pour le Néolithique). Cette dernière hypothèse est pour l'heure quasiment gratuite. En outre, les arguments stratigraphiques de qualité manquent pour pouvoir en discuter sereinement. Cependant, cette influence réciproque des deux "mondes" ne serait pas un cas isolé, loin s'en faut (cf. par exemple : Juan-Cabanilles, 1990; Guilaine et al., 1993; Marchand, 1999; etc.).

Il reste cependant une question : comment interpréter les industries pleinement mésolithiques (à armatures asymétriques à base concave bifaciale type pointe de Bavans et autres) attestées après 5300 av. J.-C. dans l'arc jurassien (grotte du Gardon par exemple; Perrin, 2001)? Ou, si elles sont confirmées, les dates très récentes de la baume de Montandon (Cupillard et al., 2000) ? Sur ce dernier site, l'ensemble du système économique est mésolithique (ibid. - recherches en cours de L. Chaix et P. Chiquet pour la grotte du Gardon). Cela implique la survivance de populations mésolithiques jusqu'à l'extrême fin du VI ${ }^{\mathrm{e}}$ millénaire, voire légèrement au-delà. Cette ultime phase du Mésolithique pourrait s'exprimer dans le nord du Jura par la persistance des industries à trapèzes du Mésolithique final (baume de Montandon?), que la genèse de ceux-ci ait lieu sous influence du Néolithique ancien ou non. Dans le sud du Jura cependant (grotte du Gardon), les armatures de ces ultimes populations mésolithiques témoigneraient de la persistance ou d'un retour à des formes asymétriques antérieures. Mais, pour l'heure, les données font encore défaut pour discuter de tels modèles. 


\section{RÉFÉRENCES BIBLIOGRAPHIQUES}

AFFOLTER J. (1998) - Analyse pétrographique des roches taillées, in G. Pignat, A. Winiger et coll., Les occupations mésolithiques de l'abri du Mollendruz Abri Freymond, commune de Mont-la-Ville (VD, Suisse), Cahiers d'Archéologie romande, 72, Lausanne, p. 81-88.

AFFOLTER J. (2000) - Origine des matières premières siliceuses utilisées au Mésolithique dans le Jura, in P. Crotti dir., Méso '97, Actes de la Table Ronde "Épipaléolithique et Mésolithique”, Lausanne, 21-23 novembre 1997, Cahiers d'Archéologie Romande, 81, Lausanne, p. 49-52.

AIMÉ G. dir. (1993) - Les abris sous roche de Bavans (Doubs) Mémoire de la Société d'Agriculture, Lettres, Sciences et Arts de la Haute-Saône, Agriculture, 3, Vesoul, 461 p., 198 fig.

AIMÉ G., JEUNESSE C. (1986) - Le niveau 5 des abris sous roche de Bavans (Doubs) et la transition Mésolithique récent/Néolithique dans la moyenne vallée du Doubs, Actes $d u X^{e}$ Colloque Interrégional sur le Néolithique, Caen 30 sept. -2 oct. 1983, Revue archéologique de l'Ouest, supplément $\mathrm{n}^{\circ} 1$, p. 31-40.

BANDI H.-G. dir. (1963) - Birsmatten-Basisgrotte. Eine mittelsteinzeitliche Fundstelle im unteren Birstal, Acta Bernansia I, 271 p., 137 fig.

BINDER D. (1987) - Le Néolithique ancien provençal. Typologie et technologie des outillages lithiques, XXIV supplément à Gallia Préhistoire, Paris, CNRS, 207 p., 182 fig.

BINTZ P. (1999) - Le Mésolithique des Alpes françaises : bilan des connaissances, in A. Beeching dir., Circulations et identités culturelles alpines à la fin de la Préhistoire. Matériaux pour une étude. Programme CIRCALP 1997/1998, Travaux du Centre d'Archéologie Préhistorique de Valence, 2, Valence, Agence Rhône-Alpes pour les Sciences Humaines, p. 317-329

BINTZ P., PICAVET R., ÉVIN J. (1995) - L'évolution culturelle du Mésolithique au Néolithique moyen en Vercors et dans les Alpes du nord, in J.-L. Voruz dir., Chronologies néolithiques. De 6000 à 2000 avant notre ère dans le Bassin rhodanien, Actes du Colloque d'Ambérieu-en-Bugey, 19-20 septembre 1992, Ambérieu-en-Bugey, Document du Département d'Anthropologie et d'Ecologie de l'Université de Genève, 20, Société préhistorique rhodanienne, p. 41-53.

CLARK J.G.D. (1958) - Blade and trapeze industries of the European Stone Age, Proceedings of the Prehistoric Society, p. 24-42.

CUPILLARD C. (1998a) - L'abri des Cabônes à Ranchot (Jura), in C. Cupillard et A. Richard dir., Les derniers chasseurs-cueilleurs du Massif jurassien et ses marges (13000-5500 avant Jésus-Christ), Lons-le-Saunier, Centre Jurassien du Patrimoine, p. 112-113.

CUPILLARD C. (1998b) - Matières premières siliceuses et territoires d'approvisionnement, in C. Cupillard et A. Richard dir., Les derniers chasseurs-cueilleurs du Massif jurassien et ses marges (13000-5500 avant Jésus-Christ), Lons-le-Saunier, Centre Jurassien du Patrimoine, p. $153-156$.

CUPILLARD C., CHAIX L., PININGRE J.-F. (2000) - Les occupations mésolithiques de la grotte de la baume de Montandon à Saint Hippolyte (Doubs, France), Actes du colloque international de Besançon, Les derniers chasseurs-cueilleurs d'Europe occidentale octobre 1998, Besançon, Presses Universitaires Franc-Comtoises, p. 219-251.

DECORMEILLE A., HINOUT J. (1982) - Mise en évidence des différentes cultures mésolithiques dans le Bassin parisien par l'analyse des données, Bulletin de la Société préhistorique française, t. $79, \mathrm{n}^{\circ} 3$, p. $81-88$.

DESBROSSE R., PARRIAT H., PERRAUD R. (1961) - La grotte du Souhait à Montagnieu (Ain), La Physiophile, ${ }^{\circ}$ 54, p. 3-68.

FRELIN-KHATIB C., THÉVENIN A. (2000) - Le Mésolithique du département de l'Ain, in P. Crotti dir., Méso '97, Actes de la Table Ronde "Épipaléolithique et Mésolithique", Lausanne, 21-23 novembre 1997, Cahiers d'Archéologie romande, 81, Lausanne, p. 155-164.

GEHLEN B. (1992) - Die Grabung in der Burghöhle Dietfurt, Gemeinde Inzigkofen-Vislingen, Kreis Sigmaringen, Archäologische Ausgrabungen im Baden Württemberg, p. 50-56.
GOB A. (1976) - La notion de "style de débitage" peut-elle servir de repère chronologique dans le Mésolithique? Congrès Préhistorique de France, $X X^{e}$ session, Provence 1974, Paris, Société préhistorique française, p. 311-319

GUILAINE J. et al., (1993) - Dourgne. Derniers chasseurs-collecteurs et premiers éleveurs de la haute vallée de l'Aude, Carcassonne, Centre d'Anthropologie des Sociétés Rurales, Toulouse, Archéologie en Terre d'Aude, 501 p.

HINOUT J. (1984) - Les outils et armatures-standards mésolithiques dans le Bassin parisien par l'analyse des données, Actes du $9^{\circ} \mathrm{Col}$ loque Interrégional sur le Néolithique, Le Néolithique dans le Nord de la France et le Bassin parisien, Compiègne, 24-26 septembre 1982, Revue archéologique de Picardie, $\mathrm{n}^{\circ}$ 1-2, p. 9-30.

HOFMANN-WYSS A.B. (1978) - Liesbergmühle VI. Eine mittelsteinzeitliche Abristation im Birstal, Schriften des Seminars für Urgeschichte der Universität Bern, Heft 2, Bern, 109 p., ill., 28 pl.

JEUNESSE C. (1986) - Rapports avec le Néolithique ancien d'Alsace de la céramique "danubienne" de la Hoguette (à Fontenay-leMarmion, Calvados), Actes du $X^{e}$ Colloque Interrégional sur le Néolithique, Caen 30 sept.-2 oct. 1983, Revue archéologique de l'Ouest, supplément $\mathrm{n}^{\circ} 1$, p. 41-50.

JEUNESSE C. (1987) - La céramique de la Hoguette. Un nouvel "élément non rubané" du Néolithique ancien de l'Europe du nord-ouest, Cahiers alsaciens d'Archéologie, d'Art et d'Histoire, t. XXX, p. 5-33.

JEUNESSE C. (1998) - La néolithisation de l'Europe occidentale (VII'$\mathrm{V}^{\mathrm{e}}$ millénaire) : nouvelles perspectives, in $\mathrm{C}$. Cupillard et A. Richard dir., Les derniers chasseurs-cueilleurs du Massif jurassien et ses marges (13000-5500 avant Jésus-Christ), Lons-le-Saunier, Centre Jurassien du Patrimoine, p. 208-217.

JUAN-CABANILLES J. (1990) - Substrat épipaléolithique et néolithisation en Espagne : apport des industries lithiques à l'identification des traditions culturelles, in D. Cahen et M. Otte dir., Rubané et Cardial, Actes du colloque de Liège, novembre 1988, ERAUL n 39 , Liège, p. 417-435.

LAPLACE G. (1964) - Essai de typologie systématique, Annali del'Università di Ferrara (Nuova Serie), Sezione XV, Paleontologia umana e paletnologia, Supplemento II al volume I, 85 p., 8 fig.

LÖHR H. (1991) - La latéralisation des armatures asymétriques à la charnière Mésolithique-Néolithique, Actes de la table ronde de Beaufort, Épipaléolithique et Mésolithique entre Ardennes et Massif alpin, 17 et 18 novembre 1990, Bulletin de la Société préhistorique luxembourgeoise, t. 12 , p. 53-64.

MANEN C. (1997) - L'axe rhodano-jurassien dans le problème des relations sud-nord au Néolithique ancien, Oxford, BAR International Series 665,233 p., 137 fig.

MARCHAND G. (1999) - La Néolithisation de l'ouest de la France. Caractérisation des industries lithiques, Oxford, BAR International Series 748, 381 p., 106 fig., 105 pl.

MAZURIÉ DE KEROUALIN K. (2000) - La première néolithisation de l'Europe : une réévaluation des modalités de peuplement, Thèse de troisième cycle, Université de Genève, Département d'Anthropologie et d'Écologie, inédit, 499 p., fig., annexes.

MORELON S. (1973) - Le gisement préhistorique de "La Touvière", commune d'Arbignieu (Ain), Documents des Laboratoires de Géologie de la Faculté des Sciences de Lyon, ${ }^{\circ}$ 56, p. 1-165.

PERRIN T. (2001) - Évolution du silex taillé dans le Néolithique hautrhodanien autour de la stratigraphie du Gardon (Ambérieu-en-Bugey, Ain), Thèse de doctorat, Université de Paris I Panthéon-Sorbonne, inédit, 3 vol., 423 et 346 p., 270 fig., 218 pl.

PERRIN T. (à paraitre) - Industries lithiques taillées et groupes culturels dans le haut Bassin rhodanien au Néolithique ancien, Actes des Quatrièmes Rencontres Méridionales de Préhistoire Récente, Nîmes, 28 et 29 octobre 2000. 
PICAVET R. (1991) - L'abri sous roche de la Grande Rivoire (Sassenage, Isère), Mémoire de Diplôme, École des Hautes Études en Sciences Sociales, Toulouse, inédit, 219 p., 94 fig.

PIGNAT G., WINIGER A. et coll. (1998) - Les occupations mésolithiques de l'abri du Mollendruz. Abri Freymond, commune de Mont-laVille (VD, Suisse), Cahiers d'Archéologie romande, 72, Lausanne, 212 p., 220 fig., $16 \mathrm{pl}$.

POUSAZ N. et al. (1991) - L'abri sous roche mésolithique des Gripons à Saint-Ursanne (JU/Suisse), Cahier d'Archéologie jurassienne, 2, Porentruy, 176 p., 126 fig., $12 \mathrm{pl}$.

ROZOY J.-G. (1968) - L'étude du matériel brut et des microburins dans l'Épipaléolithique (Mésolithique) franco-belge, Bulletin de la Société préhistorique française, t. LXV, Etudes et Travaux, 1, p. 365-390.

ROZOY J.-G. (1978) - Les derniers chasseurs : l'Épipaléolithique en France et en Belgique, essai de synthèse, Numéro spécial juin 1978 du Bulletin de la Société archéologique champenoise, Charleville, 3 vol., 1257 p., 290 fig., 258 pl.

SABATIER P. (1997) - Représentation d'ensembles de dates radiocarbones sous forme d'histogrammes pondérés. La vallée du Rhône de 6000 à 2000 avant notre ère, Mémoire de DEA, EHESS Toulouse, dactylographié, inédit, 144 p., 26 fig.

SAINTY J., THÉVENIN A. (1998) - L'abri du Mannlefelsen I à Oberlarg (Haut-Rhin), in C. Cupillard et A. Richard dir., Les derniers chasseurs-cueilleurs du Massif jurassien et ses marges (13000-5500 avant Jésus-Christ), Lons-le-Saunier, Centre Jurassien du Patrimoine, p. 122-123.

SCHÜTZ C., STRIEN H.-C., TAUTE W., TILLMANN A. (1991) Ausgrabungen in der Wilhelma von Stuttgart-Bad Cannstatt : die erste Siedlung der altneolithischen la-Hoguette-Kultur, Archäologische Ausgrabungen im Baden Württemberg, p. 45-49.

SÉARA F. (1998) - Principes et évolution du débitage à partir des séries lithiques de Choisey et de Ruffey-sur-Seille (Jura), in C. Cupillard et A. Richard dir., Les derniers chasseurs-cueilleurs du Massifjurassien et ses marges (13000-5500 avant Jésus-Christ), Lons-le-Saunier, Centre Jurassien du Patrimoine, p. 150-152.

SEARAF. (2000) - Approche de l'organisation spatiale de campement de chasseurs-cueilleurs mésolithiques : le cas de Ruffey-sur-Seille dans le Jura (France), in P. Crotti dir., Méso '97, Actes de la Table Ronde "Épipaléolithique et Mésolithique", Lausanne, 21-23 novembre 1997, Cahiers d'Archéologie romande, 81, Lausanne, p. $139-150$

THEVENIN A. (1990) - Du Dryas III au début de l'Atlantique : pou une approche méthodologique des industries et des territoires dans
l'Est de la France (1 $1^{\text {re }}$ partie), Revue archéologique de l'Est et du Centre-Est, t. 41, fasc. 2, p. 177-212.

THÉVENIN A. (1991) - Du Dryas III au début de l'Atlantique : pour une approche méthodologique des industries et des territoires dans l'Est de la France ( $2^{\mathrm{e}}$ partie), Revue archéologique de l'Est et du Centre-Est, t. 42, fasc. 1, p. 4-62.

THÉVENIN A. (1995) - Mésolithique récent, Mésolithique final, Néolithique ancien dans le nord-est de la France : pour une réinterprétation des données, Revue archéologique de Picardie, $\mathrm{n}^{\circ}$ spéc. 9, p. 3-15.

THÉVENIN A. (1998) - Le Mésolithique du Centre-Est de la France : chronologie, peuplement, processus évolutifs, Revue archéologique de l'Est, t. 49, p. 87-133.

THÉVENIN A., SAINTY J. (1974) - Préhistoire en Alsace : AchenheimOberlarg ou 600000 ans de Préhistoire, Archeologia, $n^{\circ} 75$, p. 49-61

VAN BERG P.-L. (1991) - La céramique néolithique ancienne non rubanée dans le nord-ouest de l'Europe, Bulletin de la Société préhistorique luxembourgeoise, t. 12, p. 107-124.

VILAIN R. (1966) - Le gisement de Sous-Balme à Culoz (Ain) et ses industries microlithiques, Documents des Laboratoires de Géologie de la Faculté des Sciences de Lyon, n 13, 219 p., 45 fig., 23 pl.

VORUZ J.-L. (1999) - Chronologie de la néolithisation dans le hau Bassin rhodanien, in J. Evin, C. Oberlin, J.-P. Daugas et J.-F. Salles dir., ${ }^{14}$ Cet Archéologie, Actes du $3^{2}$ Congrès International, Lyon, 6-10 avril 1998, Mémoire de la Société préhistorique française, t. XXVI, et supplément de la Revue d'Archéométrie, p. 461-464.

WALCZAK J. (1998) - La question des styles techniques durant le Mésolithique : remarques générales sur le style tardenoisien de Coincy et sa "valeur humaine", Bulletin de la Société préhistorique française, t. $95, \mathrm{n}^{\circ} 2$, p. $203-220$.

WITTIG M. (1997) - L'abri du Roseau à Neuville-sur-Ain (Ain), Diplôme en Archéologie Préhistorique, Université de Genève, Faculté des Sciences, Département d'Anthropologie et d'Écologie, 146 p., 45 fig.

WYSS R. dir. (1979) - Das mittelsteinzeitliche Hirschjägerlager von Schötz 7 im Wauwilermoss, Archeologische Forschungen, 111 p.

Thomas PERRIN

ATER Collège de France Centre d'Anthropologie 39, allées Jules-Guesde, 31000 Toulouse tperrin@free.fr 
